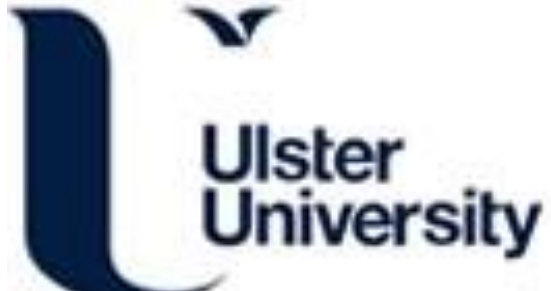

\section{miR-210 is induced by hypoxia and regulates neural cell adhesion molecule in prostate cells}

Angel, C. Z., Lynch, S. M., Nesbitt, H., McKenna, M. M., Walsh, CP., \& McKenna, D. J. (2020). miR-210 is induced by hypoxia and regulates neural cell adhesion molecule in prostate cells. Journal of Cellular Physiology, 235(9), 6194-6203. https://doi.org/10.1002/jcp.29548

Link to publication record in Ulster University Research Portal

\section{Published in:}

Journal of Cellular Physiology

Publication Status:

Published (in print/issue): 09/06/2020

DOI:

10.1002/jcp.29548

\section{Document Version}

Author Accepted version

\section{General rights}

Copyright for the publications made accessible via Ulster University's Research Portal is retained by the author(s) and / or other copyright owners and it is a condition of accessing these publications that users recognise and abide by the legal requirements associated with these rights.

\section{Take down policy}

The Research Portal is Ulster University's institutional repository that provides access to Ulster's research outputs. Every effort has been made to ensure that content in the Research Portal does not infringe any person's rights, or applicable UK laws. If you discover content in the Research Portal that you believe breaches copyright or violates any law, please contact pure-support@ulster.ac.uk. 
Title: miR-210 is Induced by Hypoxia and Regulates Neural Cell Adhesion Molecule in Prostate Cells

Running Title: Role of hypoxia-induced miR-210 in prostate cancer

Authors : Charlotte Zoe Angel ${ }^{1}$, Seodhna M. Lynch ${ }^{2}$, Heather Nesbitt ${ }^{1}$, Michael M. McKenna ${ }^{3}$, Colum P. Walsh ${ }^{1}$, Declan J. McKenna ${ }^{1 *}$

\section{Affiliations:}

${ }^{1}$ Biomedical Sciences Research Institute Ulster University

Cromore Road

Coleraine

BT52 1SA

UK

${ }^{2}$ School of Biomolecular and Biomedical Science,

Conway Institute

University College Dublin

Dublin 4

IRELAND

${ }^{3}$ Department of Cellular Pathology

Western Health \& Social Care Trust

Altnagelvin Area Hospital

Glenshane Road, Co. Derry

BT47 6SB

UK

* Corresponding author:

Dr Declan McKenna

Biomedical Sciences Research Institute

University of Ulster

Coleraine

BT52 1SA

UK

Tel. +44(0)28 70124356

Email.dj.mckenna@ulster.ac.uk

\section{ACKNOWLEDGEMENTS}

Funding for this research was provided by a PhD Studentship Award from the Department for the Economy (DfE), Northern Ireland.

\section{CONFLICT OF INTEREST STATEMENT}

The authors declare no competing interests. 


\section{ABSTRACT}

Hypoxia in prostate tumours has been associated with disease progression and metastasis. MicroRNAs are short non-coding RNA molecules which are important in several cell processes, but their role in hypoxic signalling is still poorly understood. miR-210 has been linked with hypoxic mechanisms, but this relationship has been poorly characterised in prostate cancer. In this report the link between hypoxia and miR-210 in prostate cancer cells is investigated.

PCR analysis demonstrates that miR-210 is induced by hypoxia in prostate cancer cells using in vitro cell models and an in vivo prostate tumour xenograft model. Analysis of The Cancer Genome Atlas (TCGA) prostate biopsy datasets shows that miR-210 is significantly correlated with Gleason grade and other clinical markers of prostate cancer progression. NCAM is identified as a target of miR-210, providing a biological mechanism whereby hypoxia-induced miR-210 expression can contribute to prostate cancer.

This study provides evidence that miR-210 is an important regulator of cell response to hypoxic stress and proposes that its regulation of NCAM may play an important role in the pathogenesis of prostate cancer.

KEYWORDS: hypoxia, microRNA, miR-210, prostate cancer, NCAM 


\section{INTRODUCTION}

It is well established that hypoxia, defined as a level of oxygen tension below the normal physiologic level, occurs in most solid tumours (McKeown, 2014; Muz et al, 2015). Hypoxia develops in tumours due to the deregulated proliferation of cancer cells away from their blood supply and the increasingly chaotic and leaky structure of tumour vasculature (Fraga et al, 2015). This is an important factor in cancer progression because hypoxic stress triggers a complex network of cellular and molecular responses that can promote tumour growth (Muz et al, 2015; Araos et al, 2018). This is particularly significant for prostate tumours which are known to be very hypoxic in comparison to normal prostate tissue (McKeown et al, 2014; McKenna et al, 2018). Prostate tumour hypoxia has been implicated as a contributory factor in malignant progression (Rudolsson et al, 2009; Tsai \& Wu, 2012), genetic instability (Taiakina et al, 2014), epithelial-to-mesenchymal transition (EMT)(Byrne et al, 2016; Li et al, 2016) and the selection of cells with diminished apoptotic potential and a greater invasive potential (Butterworth et al, 2008; Graeber et al, 1996). However, although many of the biological pathways involved in the hypoxic response have been investigated (Muz et al, 2015), the role of microRNAs (miRNAs) is only beginning to emerge and be understood.

miRNAs are short, non-coding RNAs that direct the degradation or translational inhibition of messenger RNAs (mRNAs). miRNAs are known to regulate many essential cell processes including the cell cycle, apoptosis, metabolism, differentiation, and immunosuppression, as well as facilitating local and systemic interactions by being transmitted in vesicles such as exosomes and in bodily fluids (Hayes et al, 2014). It is therefore unsurprising that the abnormal expression of individual miRNAs is associated with many diseases, including cancer (lorio \& Croce, 2012). In prostate cancer, several miRNAs are expressed aberrantly, but the precise ways in which they contribute to the development and progression of cancer remains to be fully explained (Sharma \& Baruah, 2019; Kanwal et al, 2017). Moreover, few microRNAs have been specifically investigated in relation to hypoxia in prostate cancer.

One miRNA that has been consistently linked with hypoxia in various tissues is hsa-miR-210-3p (miR210), to the extent that it is now considered a major regulator of the hypoxic response (Bavelloni et al, 2017). Many studies have shown how miR-210 contributes to several important cell processes, including angiogenesis (Lin et al, 2018), DNA damage response (Crosby et al, 2009), cell proliferation and apoptosis (Zhang et al, 2015), through regulation of key gene targets. Hence, abnormal expression 
of miR-210 can impact dramatically on cell behaviour, with a large body of evidence now gathered which links it with cancer development (Bavelloni et al, 2017; Dang \& Myers, 2015). For example, overexpression of miR-210 has been associated with breast (Li et al, 2013; Madhavan et al, 2012; Camps et al, 2008), renal (Petrozza et al, 2017), lung (He et al, 2018), colorectal (Sabry et al, 2018) and pancreatic (Greither et al, 2010) cancers among others. Together, these studies indicate a vital contributory role for miR-210 in cancer development, as well as demonstrating its potential value as a prognostic cancer marker (Liu et al, 2017). Given its well-established role as a hypoxic regulator, it seems reasonable to presume that the miR-210 over-expression is a reflection of the hypoxia that occurs in most tumours.

However, even though prostate tumours are known to be very hypoxic, it appears that only a few studies have investigated a role for miR-210 in prostate cancer. Of those that have, recent studies have found that over-expression of miR-210 in primary prostate tumours has been shown to correlate with lymphogenic metastatic (Eminaga et al, 2018) and bone metastatic (Dai et al, 2017; Ren et al, 2017) disease. Likewise, sera from patients with prostate cancer have higher levels of miR-210 (Qu \& Huang, 2018; Cheng et al, 2013). In vitro work has shown that miR-210 targets key players in the NF-KB (Ren et al, 2017) and TGF- $\beta$ (Dai et al, 2017) signaling pathways, while others have suggested miR-210 expression in stromal fibroblasts can contribute to prostate tumour progression (Taddei et al, 2014; Andersen et al, 2016) through promotion of EMT (Taddei et al, 2014). However, none of these studies have specifically examined the link between hypoxia and miR-210 in prostate cancer. Thus, there is still a clear need to study miR-210 to improve understanding about how hypoxia can drive prostate cancer. Therefore, in this study, we investigated the expression and regulatory mechanisms of miR-210 in response to hypoxia in prostate cancer cells. 


\section{METHODS}

Cell culture and transfections. All cell-lines were obtained from American Type Culture Collection (ATCC, Rockville, MD, USA). Cells were frozen at low passage number and used within 3-6 passages after thawing. Cells were authenticated by in-house genotyping service and routinely tested as mycoplasma-free (InvivoGen, Toulouse, France). Non-malignant prostate epithelial cell-line RWPE1 was cultured in keratinocyte growth medium supplemented with $5 \mathrm{ng} / \mathrm{ml}$ human recombinant epidermal growth factor and $0.05 \mathrm{mg} / \mathrm{ml}$ bovine pituitary extract (Life Technologies, Paisley, UK). Human prostate cancer cell-lines LNCaP, 22RV1 and PC3 were cultured in RPMI-1640 supplemented with 10\% FBS and L-glutamine (Life Technologies). All cells were grown in an incubator with a humidified atmosphere of $95 \%$ air and $5 \% \mathrm{CO} 2$ at $37^{\circ} \mathrm{C}$ and routinely passaged. For treatment in hypoxic conditions, cells were placed in normoxia ( $20 \%$ oxygen) or hypoxia $\left(0.1 \%\right.$ oxygen) at $37^{\circ} \mathrm{C}$ in a hypoxia work station (Ruskinn Technology, UK) for up to 72 hours. For spheroid cell culture, 6-well plates were double-coated with a polyhema acrylamide layer (1.2\% poly(2-hydroxyethyl methacrylate) in $95 \%$ ethanol) to prevent cell adhesion to the base of the plate. 30,000 LNCaP cells were seeded per well, media was replaced every 2-3 days and average spheroid size $(n=20)$ was measured by microscopy. For miRNA transfections, RWPE1 cells were seeded at 80,000 cells/well in a 6-well plate. After $24 \mathrm{hrs,} \mathrm{cells} \mathrm{were} \mathrm{transfected} \mathrm{with}$ miR-210 (pre-miR-210) or non-targeting negative control (pre-miR-neg) (both Life Technologies) at a final concentration of 25nM using Lipofectamine 2000 (Life Technologies). After 72 hours, cells were harvested for RNA or protein extraction.

Luciferase Reporter Assay. Luciferase reporter plasmid constructs were a kind gift from Dr Fabio Martelli as previously published (Fasanaro et al, 2009). One construct contained the wild-type NCAM1 3'UTR region with the miR-210 binding site intact (WT-3'UTR). A matched control construct (DEL3'UTR) contained deletions in the seed region of the miR-210 binding site. Cells were seeded at a concentration of 20,000 cells/well in 24 well plates and transfected with 300 ng of either WT-3'UTR plasmid or DEL-3'UTR, together with either pre-miR-210 or control pre-miR-neg oligonucleotides at a concentration of $25 \mathrm{nM}$. 30ng Renilla luciferase vector was included in each well to control for transfection efficiency. After 48 hours, cells were lysed in lysis buffer (Promega, Southampton, UK) and luciferase activity measured using the Dual-Glo® Luciferase Assay Kit (Promega) on a FluoStar Omega plate reader (BMG LabTech, Aylesbury, UK). Transfections were carried out in triplicate, measurements 
within experiments were performed in duplicate, and firefly luciferase readings were normalised against renilla luciferase readings before analysis.

\section{RNA Extraction from FFPE Human Prostate Tumour Samples.}

Formalin Fixed Paraffin Embedded (FFPE) prostate cancer samples were obtained from Altnagelvin Area Hospital, Derry. Use of patient material and information, as well as research protocols, were approved by ORECNNI (Ref. 10/NIR0213), which included informed consent from patients. For preparation of RNA from FFPE prostatectomy biopsy samples $(n=17)$, five $10 \mu \mathrm{M}$ sections containing $>50 \%$ tumour were cut for RNA extraction. Sections of matched normal prostate tissue from the unaffected lobe of the same patient were similarly cut. RNA extraction on all FFPE tissue was performed using the RecoverAll ${ }^{\mathrm{TM}}$ Total Nucleic Acid Isolation Kit for FFPE (Life Technologies) following manufacturer's instructions.

\section{PCR Analysis}

RNA extraction was carried out using Tripure ${ }^{\circledR}$ (Life Technologies) according to manufacturer's instructions. $1 \mu \mathrm{g}$ RNA was used for first strand cDNA synthesis using random primers with transcriptor high-fidelity cDNA synthesis kit (Roche, Sussex, UK) according to manufacturer's instructions. For quantitative Real-time PCR (qRT-PCR), amplification of PCR products was quantified using FastStart SYBR Green Master (Roche) on a Roche LC480 Lightcycler, using primer sets for NCAM1 (fw: TGCGACCATCCACCTCAAAG, rv: CCAGAGTCTTTTCTTCGCTGC), PTEN (fw: ACCCACCACAGCTAGAACTT, rv: GGGAATAGTTACTCCCTTTTTGTC), POU5F1 (fw: TGGGGGTTCTATTTGGGAAGG， rv: GATCTGCTGCAGTGTGGGT), HPRT (fw: CCTGGCGTCGTGATTAGTGA, rv: CGAGCAAGACGTTCAGTCCT). Expression was normalised to HPRT and graphs represent the combined results of three independent biological replicates. qRT-PCR of miRNAs was performed using the miRCURY LNA ${ }^{T M}$ microRNA PCR system (Exiqon, Vedbaek, Denmark). 50ng (clinical samples) or 20ng (cell-line samples) template RNA was used in each first strand cDNA synthesis reaction. PCR was performed over 40 amplification cycles and fluorescence monitored on the Roche LC480 Lightcycler. For all qRT-PCR miRNA analysis, normalisation was against U6snRNA or SNORD48 and graphs represent the combined results from 3 independent biological replicates, unless otherwise indicated. Serum miRNA was extracted using a column-based RNA isolation kit (Exiqon) using $5 \mu \mathrm{L}$ serum. miR-191 was used as housekeeping control for PCR analysis of serum miRNA expression. 


\section{Protein analysis}

Protein was extracted from spheroids using urea buffer. Primary antibodies used for blotting were antiHIF-1 $\alpha$ (Sigma, Dorset, UK), anti-NCAM (Santa Cruz Biotechnology, Heidelberg, Germany) with antia-Actin (Sigma) as loading control. Membranes were blocked in 5\% milk diluted in 1x TBS-T $(0.05 \%)$ followed by incubation in the appropriate secondary antibody (goat anti-rabbit IgG-HRP (1:10000) or goat anti-mouse IgG-HRP (1:10000))(both Santa Cruz). Luminescence was revealed by incubation with enhanced chemiluminescent reagent (Life Technologies) and signal detected on a G:BOX F3 imaging system (Syngene, Cambridge, UK).

\section{In vivo methods}

Animal maintenance and care. In vivo experiments were conducted in accordance with the Animal (Scientific Procedures) Act 1986 and the UKCCCR guidelines for the welfare of animals in experimental neoplasia (Workman et al, 1988). 8-10 week old male nude mice weighing 25-30g (Envigo, Cambridgeshire, UK) were housed under standard laboratory conditions in a temperature controlled (22ํㅜ $; 50-55 \%$ humidity) specific pathogen-free environment with a 12 -hour light/dark cycle. Food and water were supplied ad libitum. Procedures and administrations were performed using aseptic technique, and tumour implantation and oxygen electrode measurement were performed under anaesthesia. The work was approved by the ethical review committee of Ulster University and covered by establishment licence and project licence.

Xenograft establishment. LNCaP xenografts were established on nude mice by subcutaneous injection of $5 \times 10^{6}$ cells suspended in $100 \mu \mathrm{l}$ of ice-cold matrigel (Corning, growth factor reduced) to the dorsum, using a cold $21 \mathrm{~g}$ needle. Once the tumour became palpable, dimensions were measured using Vernier calipers, using the formula: volume $=($ height $\mathrm{x}$ height $\mathrm{x}$ width $) / 2$.

Oxygen electrode measurement. A fibre optic probe $(O x y L i t e \AA)$ was inserted into the tumour (avoiding proximity to blood vessels) through a $21 \mathrm{~g}$ needle. After the probe readings had normalised, 30 readings were recorded per site (the median reading was used). At least two sites (with similar readings) were measured per tumour and the mean of the 2 median readings was taken to represent the tumour. 
Drug administration. Bicalutamide (Sigma) was prepared in vehicle (0.1\% DMSO in corn oil) and administered orally via gavage at $6 \mathrm{mg} / \mathrm{kg}$ daily. When tumour volume reached between $150 \mathrm{~mm}^{3}$, mice were randomly assigned to treatment groups and dosing was initiated.

\section{Databases and online tools utilised}

To identify mRNA targets of the miRNAs, the databases miRTarBase (Chou et al, 2018) (http://mirtarbase.mbc.nctu.edu.tw) and miRWalk2.0 (Dweep \& Gretz, 2015) (http://zmf.umm.uniheidelberg.de/apps/zmf/mirwalk2/index.html) were searched to find consistently predicted targets. Some results published here are in part based upon data generated by The Cancer Genome Atlas (TCGA) Research Network: (http://cancergenome.nih.gov/). TCGA analysis was performed using Regulome Explorer (http://explorer.cancerregulome.org/) and Firebrowse (http://firebrowse.org/) analysis tools. Regulome Explorer analysis was based on a single primary prostate cancer dataset (TCGA Research Network, 2015). Firebrowse analysis was based on larger cohort of prostate adenocarcinoma (PRAD) samples (Broad Institute TCGA Centre (2016). Survival analysis was performed using both KM Express (Chen et al, 2018) (http://ec2-52-201-246-161.compute1.amazonaws.com/kmexpress/index.php) and Human Protein Atlas (Uhlen et al, 2015) (https://www.proteinatlas.org/) analysis tools. KEGG and Gene Ontology analysis was performed using DAVID Bioinformatics Resources 6.8 (Huang et al, 2009a; 2009b)(https://david.ncifcrf.gov/).

\section{Statistical analysis}

Unless otherwise indicated, all data were analysed using a two-tailed student's t-test, with Welch's correction where appropriate, using the Prism 5.0 software (GraphPad). Regulome analysis of TCGA dataset was based on All Pairs Statistical Associations and utilised Spearman's rank correlation, with p-values adjusted for multiple hypothesis testing. For Firebrowse analysis, Spearman's rank correlation and two-tailed $\mathrm{P}$ values were estimated using 'cor.test' function in R. For multiple hypothesis testing, $\mathrm{p}$ values were converted to Q-value using the Benjamini and Hochberg correction. KM Express survival analysis was performed using R package 'survival (Therneau, 2015). Differences between points were deemed statistically significant with a $p<0.05$ (95\% confidence interval). 


\section{RESULTS}

\section{Hypoxia upregulates miR-210 in prostate cancer cells.}

Growing LNCaP prostate cancer cells in hypoxic $\left(0.1 \% \mathrm{O}_{2}\right)$ conditions resulted in in significant miR-210 upregulation compared to $20 \% \mathrm{O}_{2}$ (Figure $1 \mathrm{~A}$ ). The induction of hypoxia in these cells was confirmed by upregulation of hypoxic marker genes PTEN and POU5F1 (Figure 1B) and HIF-1 $\alpha$ protein (Figure 1C). The induction of miR-210 in response to hypoxia was also noted in $22 \mathrm{RV} 1$ and PC3 prostate cancer cells, as well as normal RWPE1 epithelial prostate cells, following growth in $0.1 \% \mathrm{O}_{2}$ (Supplementary Figure 1). In LNCaP spheroid models, miR-210 expression was also increased when spheroids became hypoxic after $\sim 14$ days growth (Figures 1D $-1 F$ ). Induction of hypoxia was evidenced by induction of HIF-1 $\alpha$ protein in spheroids which had reached $\sim 50 \mu \mathrm{M}$ in size (Figure $1 \mathrm{G}$ ).

We also employed a murine LNCaP xenograft model which we have previously used to study tumour hypoxia in response to bicalutamide treatment (Byrne et al, 2016; Nesbitt et al, 2017). As expected, tumour growth was significantly inhibited by bicalutamide treatment after 28 days, in comparison to vehicle treated tumours (Figure 2A). As we previously shown in similar models, bicalutamide treatment induces profound tumour hypoxia after 7 days (Figure 2B), resulting in HIF-1 $\alpha$ stabilization (Figure 2E). An induction of miR-210 is shown which becomes significant after 28 days (Figure $2 \mathrm{C}$ ), when tumour hypoxia is still apparent. There was also an induction of miR-210 in serum at Day 7, although this fell short of significance (Figure 2D).

\section{miR-210 is associated with markers of prostate cancer progression.}

We successfully measured miR-210 levels in in 17 matched pairs of normal and tumour tissue from clinical FFPE prostate biopsies (Figure 3A). Up-regulation as well as down-regulation of miR-210 was observed in tumour tissue compared to paired normal tissue, which we suggest is indicative of the relative hypoxic status of cells within an individual tumour, which in turn may provide useful diagnostic or prognostic information. We also noted with interest that there was a trend for higher miR-210 expression as Gleason score increased in these samples, even allowing for the small number of samples (Figure 3B). When we progressed to perform similar analysis on much larger numbers of samples, using TCGA datasets, we showed that increased miR-210 expression was indeed significantly increased in tumours with higher Gleason, as well showing significant positive correlation with tumour 
stage and lymph node metastasis (Figures 3C and 3D). When cases were split into high and low miR210 expression, it was evident that high miR-210 expression resulted in significantly shorter time of disease-free progression (Figure 3E). To further evidence that the increased expression of miR-210 is associated with hypoxia in clinical samples, we examined expression of various hypoxia-related genes in a well-characterized TCGA dataset $(\mathrm{n}=330)$. This showed that $E Z H 2$, another marker of prostate tumour hypoxia (zhou et al, 2018), has a strong positive correlation with miR-210 expression in these samples (Figure 3F). Likewise, the hypoxia-induced genes SPP1 and DLGAP5, which code for ostepontin and HURP proteins respectively, also show significant positive correlation with clinicpathological parameters of prostate cancer progression (Supplementary Figure 2). Finally, KEGG analysis and Gene Ontology mapping of validated miR-210 targets demonstrates that miR-210 is significantly associated with both prostate cancer and hypoxia-related cell processes (Supplementary Tables 1 and 2). This in silico analysis corroborates our laboratory results in demonstrating that miR210 is likely to be a key mediator of the hypoxic response in prostate cancer.

\section{Neural cell adhesion molecule (NCAM) is a direct target of miR-210.}

Using bioinformatics prediction tools, we had identified the gene NCAM1 as a potential target of miR210 (Figure 4A), so we proceeded to investigate how its expression correlated with miR-210 in prostate cancer. To test this relationship in vitro, we tested prostate cell-lines to identify one that expressed detectable levels of NCAM protein (Figure 4B), and subsequently selected RWPE1 cells in which to over-express miR-210 by transient transfection (Figure 4C). NCAM1 levels as measured by qPCR were decreased, although this was not significant, probably because the mRNA is incompletely degraded by the binding and can still be amplified, albeit to a reduced extent (Figure 4D). However, at the protein level there was a marked reduction in NCAM levels, suggesting miR-210 can indeed regulate levels of functional NCAM (Figure 4E). We confirmed that NCAM1 was a direct target of miR-210 with a luciferase reporter assay (Figure 4F). The luciferase activity of a reporter construct containing the wildtype NCAM1 3'UTR region (WT-3'UTR) showed significant reduction when miR-210 was overexpressed in the same cells. However, when miR-210 was over-expressed with a reporter construct which had deleted residues in the miR-210 binding site of NCAM1 3'UTR (DEL-3'UTR), no reduction in luciferase activity was observed, therefore indicating that NCAM1 is a direct target of miR-210 in these cells. Hence, through this mechanism, the hypoxia-induced expression of miR-210 has the potential to impact upon cell adhesion processes. 


\section{miR-210 and NCAM expression is inversely correlated in prostate tissue}

We hypothesised that increased hypoxia in prostate epithelial cells and tumours induces miR-210 which in turn decreases the levels of NCAM1, so we explored TCGA datasets to investigate this relationship. We found that a significant inverse correlation does indeed exist between miR-210 and NCAM1 gene expression in prostate biopsy tissue (Figure 5A). Expression of NCAM1 also showed a significant negative correlation with clinicopathological markers of prostate cancer progression (Figure 5B), in contrast to the positive correlation observed for miR-210 (Figure 3C). As an illustrative example, NCAM1 expression is higher in tumours with Gleason Score $\leq 7$, compared to those with Gleason score $\geq 8$ (Figure 5C), whereas the opposite is true for miR-210 (Figure 3D). Survival analysis indicates that NCAM1 expression is associated with lower survival probability at $>10$ years, but this association falls short of significance, so NCAM1 expression by itself it would not be considered a reliable prognostic factor in prostate cancer (Supplementary Figure 3A). 


\section{DISCUSSION}

Prostate tumours are recognised as being very hypoxic, so it is surprising that few studies have specifically investigated how this impacts upon miR-210, a microRNA which has been consistently linked with the hypoxic response. Hence, this is the first report to present research showing how the relationship between tumour hypoxia and miR-210 can contribute to prostate cancer development through its regulation of NCAM levels.

We have provided evidence that miR-210 is induced by hypoxia in in vitro and in vivo models of prostate cancer. In our previous in vivo work we demonstrated how hypoxia can be induced in a xenograft tumour model by bicalutamide, a phenomenon which we proposed may drive the metastatic and invasive potential of prostate tumour cells (Byrne et al, 2016; Nesbitt et al, 2017). Here, we show that the hypoxic stress up-regulates miR-210, which will in turn influence a number of genes and cell processes linked to tumour growth. The analysis of clinical biopsy samples presented here also supports the link between the hypoxic nature of prostate tumours, increased expression of miR-210 and prostate cancer progression. It is also interesting to note that increased miR-210 expression decreases time of diseasefree survival. This substantiates our previous findings which suggested increased tumour hypoxia was a key contributory factor in prostate cancer relapse. Taken together, it therefore seems clear that the hypoxia which is well-known to exist in prostate tumours does result in over-expression of miR-210, which in turn can cause cellular dysfunction through its impact upon many biological pathways.

To explore potential novel mechanisms of miR-210 action, we progressed to analyse its effect on the translation of neural cell adhesion molecule (NCAM), a large cell surface receptor from the immunoglobin superfamily. NCAM, also known as CD56, is classically known as a cell surface molecule which plays an important role in neurons (Aonurm-Helm et al, 2016), haematopoietic stem cells (Simmons et al, 2001) and immune cells (Van Acker et al, 2017). It is encoded by the gene NCAM1 and three splice variants exist, giving rise to proteins of approximately $180 \mathrm{kDa}, 140 \mathrm{kDa}$ or $120 \mathrm{kDa}$ in weight (Aonurm-Helm et al, 2016). These facilitate interactions with other cells through both homophillic NCAM binding and heterophillic binding with other molecules, such as fibroblast growth factor receptor (FGFR) on the surface of T cells (Kos \& Chin, 2002). However NCAM is also involved in many other roles in other cell types including exon guidance and repair, migration, apoptosis and cell proliferation (Jensen \& Berthold, 2007). In cancer, its role appears to be context-dependent. Neoplasms of 
neuroendocrine or hematopoietic origins over-produce NCAM, as do brain tumours (Jensen \& Berthold, 2007; Zecchini \& Cavallaro, 2010). Conversely, in pancreatic (Fogar et al, 1997), colorectal (Roesler et al, 1997) astrocytic (Sasaki et al, 1998) and thyroid (Muthusamy et al, 2018; Pyo et al, 2018) cancer, low levels of NCAM have been correlated with increased malignancy. In prostate cancer, however, the role of NCAM has not been investigated.

In this study we have demonstrated that miR-210 targets NCAM1 in prostate cells. Only one previous study had identified NCAM1 as a target of miR-210 (Fasanaro et al, 2009), but ours is the first study to demonstrate this interaction may be important in a cancer setting. Interestingly, we found that none of the NCAM protein isoforms were detectable in cancer cell-lines, so we conducted these experiments in RWPE1 cells where the $120 \mathrm{kDa}$ variant was found at detectable levels. The absence of NCAM protein expression in prostate cancer cell lines suggests that its loss might be important in the carcinogenesis of prostatic epithelial cells. Indeed, our analysis of TCGA data demonstrates that low expression of NCAM1 is associated with markers of aggressiveness. Our survival analysis in prostate cancer did not show a significant correlation for NCAM1, but it is worth noting that low expression of NCAM1 is significantly associated with lower 5-year survival probability in pancreatic cancer (Supplementary Fig 3B). Given these findings, we propose that induction of miR-210 can leads to loss of NCAM which could potentially promote tumour growth in two ways. First, it would allow cells to evade immune response by decreasing the cell-cell interaction with CD56+ NK cells, FGFR+ T cells, or macrophages. Second, the loss of NCAM binding between with neighbouring cells and the extracellular matrix (ECM) could allow prostate cells to detach from their location and spread. In addition, miR-210 will also impact upon cell behaviour through the rest of its regulatory network (Supplementary Figure 4).

In summary, this is the first study to show that hypoxia-induced expression of miR-210 in prostate cells regulates NCAM expression. We have shown that miR-210 is significantly upregulated by hypoxia in prostate cells in vitro and in vivo, as well as demonstrating that miR-210 expression is positively correlated with tumour aggressiveness in human prostate cancer tissue. This study provides evidence for the use of miR-210 as a diagnostic or prognostic marker in prostate cancer, and may also be a potential target for therapeutic intervention. 


\section{DATA AVALABILITY STATEMENT}

All data generated or analysed during this study are included in this published article (and its Supplementary Information files). The data that support the findings of this study are available in The Cancer Genome Atlas at https://www.cancer.gov/about-nci/organization/ccg/research/structuralgenomics/tcga (PRAD datasets). Full details are provided in methods section and these data were derived from the following resources available in the public domain:

TCGA Research Network. DOI: https://doi.org/10.1016/i.cell.2015.10.025

Broad Institute http://gdac.broadinstitute.org/runs/stddata 20160128

\section{COMPETING INTERESTS}

The authors declare no competing interests.

\section{AUTHOR CONTRIBUTIONS}

CZA and DJM designed the study, collected the samples, performed the experiments, analyzed data, interpreted the results, and wrote the manuscript. SML and HN collected samples and performed animal experiments. MMM contributed expertise in the collection of the clinical samples. CPW secured the ethical approval for their use in this study and contributed to writing of manuscript. All authors reviewed the manuscript. 


\section{REFERENCES}

Andersen, S. et al. (2016). Fibroblast miR-210 overexpression is independently associated with clinical failure in Prostate Cancer - a multicenter (in situ hybridization) study. Sci. Rep. 6, 36573.

Aonurm-Helm, A., Jaako, K., Jürgenson, M., Zharkovsky, A. (2016). Pharmacological approach for targeting dysfunctional brain plasticity: Focus on neural cell adhesion molecule (NCAM). Pharmacol. Res. 113, 731-738.

Araos, J., Sleeman, J.P., Garvalov, B.K. (2018). The role of hypoxic signalling in metastasis: towards translating knowledge of basic biology into novel anti-tumour strategies. Clin. Exp. Metastasis 35, 563599.

Bavelloni, A. et al. (2017). MiRNA-210: A Current Overview. Anticancer Res. 37, 6511-6521.

Butterworth, K.T. et al. (2008). Hypoxia selects for androgen independent LNCaP cells with a more malignant geno- and phenotype. Int. J. Cancer 123, 760-768.

Byrne, N.M., Nesbitt, H., Ming, L., McKeown, S.R., Worthington, J., McKenna, D.J. (2016). Androgen deprivation in LNCaP prostate tumour xenografts induces vascular changes and hypoxic stress, resulting in promotion of epithelial-to-mesenchymal transition. Br. J. Cancer 114, 659-668.

Camps, C. et al. (2008). hsa-miR-210 Is Induced by Hypoxia and Is an Independent Prognostic Factor in Breast Cancer. Clin. Cancer Res. 14, 1340-1348.

Chen, X., Miao, Z., Divate, M., Zhao, Z., Cheung, E. (2018).KM-express: an integrated online patient survival and gene expression analysis tool for the identification and functional characterization of prognostic markers in breast and prostate cancers. Database doi: 10.1093/database/bay069.

Cheng, H.H. et al. (2013). Circulating microRNA Profiling Identifies a Subset of Metastatic Prostate Cancer Patients with Evidence of Cancer-Associated Hypoxia. PLoS One 8, e69239.

Chou, C.-H. et al. (2018). miRTarBase update 2018: a resource for experimentally validated microRNA-target interactions. Nucleic Acids Res. 46, D296-302.

Crosby, M.E., Kulshreshtha, R., Ivan, M., Glazer, P.M. (2009). MicroRNA Regulation of DNA Repair Gene Expression in Hypoxic Stress. Cancer Res. 69, 1221-1229. 
Dai, Y. et al. (2017). The TGF- $\beta$ signalling negative regulator PICK1 represses prostate cancer metastasis to bone. Br. J. Cancer 117, 685-694.

Dang, K., Myers, K. (2015). The Role of Hypoxia-Induced miR-210 in Cancer Progression. Int. J. Mol. Sci. $16,6353-6372$.

Dweep, H., Gretz, N. (2015). miRWalk2.0: a comprehensive atlas of microRNA-target interactions. Nat. Methods 12, 697-697.

Eminaga, O. et al. (2018). The upregulation of hypoxia-related miRNA 210 in primary tumor of lymphogenic metastatic prostate cancer. Epigenomics 10, 1347-1359.

Fasanaro, P. et al. (2009). An integrated approach for experimental target identification of hypoxiainduced miR-210. J. Biol. Chem. 284, 35134-35143.

Fogar, P.et al. (1997). Neural cell adhesion molecule (N-CAM) in gastrointestinal neoplasias. Anticancer Res. 17, 1227-1230.

Fraga, A., Ribeiro, R., Príncipe, P., Lopes, C., Medeiros, R. (2015). Hypoxia and Prostate Cancer Aggressiveness: A Tale With Many Endings. Clin. Genitourin. Cancer 13, 295-301.

Graeber, T.G. et al. (1996). Hypoxia-mediated selection of cells with diminished apoptotic potential in solid tumours. Nature 379, 88-91.

Greither, T., Grochola, L.F., Udelnow, A., Lautenschläger, C., Würl, P., Taubert, H. (2010). Elevated expression of microRNAs 155, 203, 210 and 222 in pancreatic tumors is associated with poorer survival. Int. J. Cancer 126, 73-80.

Hayes, J., Peruzzi, P.P., Lawler, S. (2014). MicroRNAs in cancer: biomarkers, functions and therapy. Trends Mol. Med. 20, 460-469.

He, R. et al. (2018). Clinical Significance of miR-210 and its Prospective Signaling Pathways in NonSmall Cell Lung Cancer: Evidence from Gene Expression Omnibus and the Cancer Genome Atlas Data Mining with 2763 Samples and Validation via Real-Time Quantitative PCR. Cell Physiol. Biochem. 46, 925-952.

Huang, D.W., Sherman, B.T., Lempicki, R.A. (2009a). Bioinformatics enrichment tools: paths toward the comprehensive functional analysis of large gene lists. Nucleic Acids Res. 37, 1-13. 
Huang, D.W., Sherman, B.T., Lempicki, R.A. (2009b). Systematic and integrative analysis of large gene lists using DAVID bioinformatics resources. Nat. Protoc. 4, 44-57.

Institute of MIT and Harvard. Broad Institute TCGA Genome Data Analysis Center (2016). Analysisready standardized TCGA data from Broad GDAC Firehose 2016_01_28 run. Dataset [Internet]. [cited 2018 Dec 2]. Available from: http://gdac.broadinstitute.org/runs/stddata 20160128 [Accessed 2602-2019].

lorio, M.V., Croce, C.M. (2012). MicroRNA dysregulation in cancer: diagnostics, monitoring and therapeutics. A comprehensive review. EMBO Mol. Med. 4, 143-159.

Jensen, M., Berthold, F. (2007). Targeting the neural cell adhesion molecule in cancer. Cancer Lett. 258, 9-21.

Kanwal, R., Plaga, A.R., Liu, X., Shukla, G.C., Gupta, S. (2017). MicroRNAs in prostate cancer: Functional role as biomarkers. Cancer Lett. 407, 9-20.

Kos, F.J., Chin, C.S. (2002). Costimulation of T cell receptor-triggered IL-2 production by Jurkat T cells via fibroblast growth factor receptor 1 upon its engagement by CD56. Immunol. Cell Biol. 80, 364-369.

Li, M. et al. (2016). Hypoxia inducible factor-1 $\alpha$-dependent epithelial to mesenchymal transition under hypoxic conditions in prostate cancer cells. Oncol. Rep. 36, 521-527.

Li, Y., Ma, X., Zhao, J., Zhang, B., Jing, Z., Liu, L. (2013). microRNA-210 as a prognostic factor in patients with breast cancer: Meta-analysis. Cancer Biomarkers 13, 471-481.

Lin, X.-J. et al. (2018). Hepatocellular Carcinoma Cell-Secreted Exosomal MicroRNA-210 Promotes Angiogenesis In Vitro and In Vivo. Mol. Ther. Nucleic Acids 11, 243-252.

Liu, Y. et al. (2017). Prognostic evaluation of microRNA-210 in various carcinomas: Evidence from 19 studies. Medicine (Baltimore) 96, e8113.

Madhavan, D. et al. (2012). Circulating miRNAs as Surrogate Markers for Circulating Tumor Cells and Prognostic Markers in Metastatic Breast Cancer. Clin. Cancer Res. 18, 5972-5982.

McKenna, D.J., Errington, R., Pors, K. (2018). Current challenges and opportunities in treating hypoxic prostate tumors. J. Cancer Metastasis Treat. 4, 11. 
McKeown, S.R. (2014).Defining normoxia, physoxia and hypoxia in tumours-implications for treatment response. Br. J. Radiol. 87, 20130676.

Muthusamy, S. et al. (2018). CD56 expression in benign and malignant thyroid lesions. Malays. J. Pathol. 40, 111-119.

Muz, B., de la Puente, P., Azab, F., Azab, A.K. (2015). The role of hypoxia in cancer progression, angiogenesis, metastasis, and resistance to therapy. Hypoxia (Auckland, NZ) 3, 83-92.

Nesbitt, H. et al. (2017).Targeting Hypoxic Prostate Tumors Using the Novel Hypoxia-Activated Prodrug OCT1002 Inhibits Expression of Genes Associated with Malignant Progression. Clin. Cancer Res. 23, 1797-1808.

Petrozza, V. et al. (2017). Secreted miR-210-3p as non-invasive biomarker in clear cell renal cell carcinoma. Oncotarget 8, 69551-69558.

Pyo, J.-S., Kim, D.-H., Yang, J. (2018).Diagnostic value of CD56 immunohistochemistry in thyroid lesions. Int. J. Biol. Markers 33, 161-167.

Qu, Y., Huang, W. (2018). Effects of microRNA-210 on the diagnosis and treatment of prostate cancer. Mol. Med. Rep. 18, 1740-1744.

Ren, D. et al. (2017). Oncogenic miR-210-3p promotes prostate cancer cell EMT and bone metastasis via NF-kB signaling pathway. Mol. Cancer 16, 117.

Roesler, J., Srivatsan, E., Moatamed, F., Peters, J., Livingston, E.H. (1997). Tumor suppressor activity of neural cell adhesion molecule in colon carcinoma. Am. J. Surg. 174, 251-257.

Rudolfsson, S.H., Bergh, A. (2009). Hypoxia drives prostate tumour progression and impairs the effectiveness of therapy, but can also promote cell death and serve as a therapeutic target. Expert Opin. Ther. Targets 13, 219-225.

Sabry, D. et al. (2018). Role of miRNA-210, miRNA-21 and miRNA-126 as diagnostic biomarkers in colorectal carcinoma: impact of HIF-1 $\alpha-$ VEGF signaling pathway. Mol. Cell. Biochem. [Epub ahead of print] doi: 10.1007/s11010-018-3462-1

Sasaki, H. et al. (1998). Expression of the neural cell adhesion molecule in astrocytic tumors: an inverse correlation with malignancy. Cancer 82, 1921-1931. 
Sharma, N., Baruah, M.M. (2019). The microRNA signatures: aberrantly expressed miRNAs in prostate cancer. Clin. Transl. Oncol. 21, 126-144.

Simmons, P.J., Levesque, J.P., Haylock, D.N. (2001). Mucin-like molecules as modulators of the survival and proliferation of primitive hematopoietic cells. Ann. N. Y. Acad. Sci. 938, 196-207.

Taddei, M.L. et al. (2014). Senescent stroma promotes prostate cancer progression: the role of miR210. Mol. Oncol. 8, 1729-1746.

Taiakina, D., Pra, A.D., Bristow, R.G. (2014).Intratumoral Hypoxia as the Genesis of Genetic Instability and Clinical Prognosis in Prostate Cancer. Adv. Exp. Med. Biol. 772, 189-204.

The Cancer Genome Atlas Research Network. The Molecular Taxonomy of Primary Prostate Cancer. (2015). Cell 163, 1011-1025.

Therneau, T. (2015). A Package for Survival Analysis in S. version 2.38. Available at: https://cran.rproject.org/web/packages/survival/index.html [Accessed 26-02-2019].

Tsai, Y.-P., Wu, K.-J. (2012). Hypoxia-regulated target genes implicated in tumor metastasis. J. Biomed. Sci. 19, 102.

Uhlen, M. et al. (2015). Tissue-based map of the human proteome. Science 347, 1260419-1260419.

Van Acker, H.H., Capsomidis, A., Smits, E.L., Van Tendeloo, V.F. (2017). CD56 in the Immune System: More Than a Marker for Cytotoxicity? Front. Immunol. 8, 892.

Workman, P. et al. (1988). UKCCCR guidelines for the welfare of animals in experimental neoplasia. Lab. Anim. 22, 195-201.

Zecchini, S., Cavallaro, U. (2010).Neural Cell Adhesion Molecule in Cancer: Expression and Mechanisms. Adv. Exp. Med. Biol. 663, 319-333.

Zhang, S., Lai, N., Liao, K., Sun, J., Lin, Y. (2015). MicroRNA-210 regulates cell proliferation and apoptosis by targeting regulator of differentiation 1 in glioblastoma cells. Folia Neuropathol. 3, 236244.

Zhou, H. et al. (2018). Attenuation of TGFBR2 expression and tumour progression in prostate cancer involve diverse hypoxia-regulated pathways. J. Exp. Clin. Cancer Res. 37, 89. 



\section{FIGURE LEGENDS}

Figure 1. Expression of miR-210 in hypoxic prostate cancer cells in vitro

(A) qRT-PCR analysis showed increased expression of miR-210 and (B) POU5F1 and PTEN in LNCaP cells exposed to hypoxia (C) Western blot analysis shows increased expression of HIF-1 $\alpha$ in LNCaP cells exposed to hypoxia. (D \& E) LNCaP spheroid growth over 15 days is associated with increase in (F) miR-210 expression and (G) HIF-1 $\alpha$ expression. PCR data was normalised to either HPRT or U6snRNA and data shown is mean \pm SE of triplicate experiments. (Student t-test $p$-values: ${ }^{*} p<0.05$, $\left.{ }^{* *} p<0.01,{ }^{* * *} p<0.001\right)$.

Figure 2. Expression of miR-210 in hypoxic prostate cancer xenograft model.

(A) Graph shows tumour growth in LNCaP-luc xenograft Nude mouse following treatment with vehicle (0.1\% DMSO in corn oil) and bicalutamide $(6 \mathrm{mg} / \mathrm{kg} / \mathrm{day})(\mathrm{n} \geq 4$ per group). (B) Oxygen levels in tumours from bicalutamide- and vehicle-treated mice ( $n \geq 4$ per group) at $0,7,14,28$ days treatment. (2way ANOVA with Bonferroni post-test $p$-values: $\left.{ }^{*} p<0.05\right)$. (C) qRT-PCR analysis of miR-210 expression in (C) excised tumours at Day 7 and Day 28 and (D) serum at Day 7. Data is mean \pm SE from triplicate experiments. (Student t-test values: $\left.{ }^{*} \mathrm{p}<0.05\right)$. (E) Western blot analysis showing increased HIF-1 $\alpha$ expression in bicalutamide-treated tumours compared to vehicle at Day 7.

Figure 3. Expression of miR-210 in clinical prostate biopsies.

(A) qRT-PCR analysis of RNA isolated from FFPE biopsy clinical specimens ( $n=17)$ showing fold change expression of miR-210 in individual tumour cases relative to matched normal tissue (B) Expression of miR-210 in biopsies with different Gleason scores. (C) Firebrowse analysis of TCGA biopsy samples showing positive correlation of miR-210 with clinicopathological parameters (D) Regulome analysis of one TCGA dataset $(n=330)$ shows miR-210 expression is increased in biopsies with Gleason score $\geq 8$. (E) KM Express survival analysis of TCGA prostate data shows significantly shorter recurrence-free survival in patients with high miR-210 expression. (F) Regulome analysis shows miR-210 is positively correlated with hypoxic marker EZH2 in TCGA dataset $(n=330)$. TCGA analyses utilised Spearman's rank correlation, with $p$-values adjusted for multiple hypothesis testing. A p-value of $<0.05$ and $Q$-value $<0.3$ was deemed significant. 


\section{Figure 4. NCAM is a direct target of miR-210}

(A) miR-210 is computationally predicted to target NCAM1 at three positions. TargetScan predicted pairing of target region in 3' UTR of NCAM1 (bottom) and miR-210 (top), with the 8mer 'seed sequence' highlighted. Letters in bold indicate the deleted region for reporter constructs. (B) Western blot analysis of NCAM protein expression in normal epithelial prostate cell lines RWPE1 and four prostate cancer cell lines. (C) Following over-expression of miR-210 in RWPE1 cells (D) qRT-PCR and (E) western blotting shows that NCAM levels are decreased. (F) Luciferase activity reporter confirms miR-210 targeting of NCAM1 in RWPE1 cells. A reporter construct containing the wild-type NCAM1 3'UTR region (WT-3'UTR) shows significant reduction of luciferase activity when co-transfected with precursor miR210 (pre-miR-210) relative to cells co-transfected with non-targeting control (pre-miR-neg). In cells transfected with a construct containing deleted residues in the miR-210 binding site of NCAM1 3'UTR (DEL-3'UTR), no reduction in luciferase activity is observed. Data in graphs is mean $\pm S E$ from triplicate experiments (Student t-test $p$-values: ${ }^{*} p<0.05,{ }^{* *} p<0.01,{ }^{* *} p<0.001$ ).

\section{Figure 5. miR-210 and NCAM1 are inversely correlated in TCGA clinical prostate samples}

(A) Regulome analysis shows miR-210 and NCAM1 are inversely correlated in TCGA clinical prostate dataset. (B) Firebrowse analysis of TCGA prostate biopsy samples showing negative correlation of NCAM1 with clinicopathological parameters. Both analyses utilised Spearman's rank correlation, with $p$-values adjusted for multiple hypothesis testing. A p-value of $<0.05$ and $Q$-value $<0.3$ was deemed significant. (C) Analysis of TCGA dataset shows NCAM1 expression is decreased in biopsies with Gleason score $\geq 8$. Data is mean \pm SE (Unpaired t test with Welch's correction, $p$-values: ${ }^{* *} p<0.01$ ).

\section{SUPPLEMENTARY MATERIAL}

File name : Angel et al miR-210 Supplementary Data J Cell Phys

File format : PDF document

Description of data : Two supplementary tables and four supplementary figures to support main manuscript. 


\section{SUPPLEMENTARY DATA FILE}

Title: miR-210 is Induced by Hypoxia and Regulates Neural Cell Adhesion Molecule in Prostate Cells

Authors : Charlotte Zoe Angel ${ }^{1}$, Seodhna M. Lynch², Heather Nesbitt ${ }^{1}$, Michael M. McKenna ${ }^{3}$, Colum P. Walsh ${ }^{1}$, Declan J. McKenna ${ }^{1 *}$

File name : Angel et al miR-210 Supplementary Data

File format : PDF document

Description of data : Two supplementary tables and 4 supplementary figures to support main manuscript.

\section{* Corresponding author:}

Dr Declan McKenna

Biomedical Sciences Research Institute

University of Ulster

Coleraine

BT52 1SA

UK

Tel. +44(0)28 70124356

Email.dj.mckenna@ulster.ac.uk 
SUPPLEMENTARY TABLE 1. Functional KEGG analysis of miR-210-3p target genes

\begin{tabular}{lccc}
\hline Disease Term & Gene Count & P-value & Benjamini \\
\hline CANCER & 38 & 0.0026 & 0.046 \\
tobacco use disorder & 33 & 0.023 & 0.91 \\
breast cancer & 11 & 0.0057 & 0.83 \\
ovarian cancer & 9 & 0.0069 & 0.76 \\
prostate cancer & 9 & 0.039 & 0.91 \\
lung cancer & 9 & 0.041 & 0.9 \\
bladder Cancer & 9 & 0.047 & 0.88 \\
\hline
\end{tabular}

Data shows highly significant association of miR-210 with cancer-related disease terms, mediated through various target genes of miR-210. P-value generated by modified Fisher Exact test. Benjamini value represents corrected $\mathrm{P}$-value for multiple hypothesis testing using the Benjamini-Hochberg method to minimise false discovery rate. 
SUPPLEMENTARY TABLE 2. Functional gene ontology analysis of miR-210-3p target genes

\begin{tabular}{lccc}
\hline Gene Ontology Term & $\begin{array}{c}\text { Gene } \\
\text { Count }\end{array}$ & P-Value & Benjamini \\
\hline cellular response to oxygen levels & 8 & 0.000092 & 0.047 \\
cellular response to oxidative stress & 9 & 0.00022 & 0.048 \\
cellular response to hypoxia & 7 & 0.00035 & 0.049 \\
cellular response to decreased oxygen levels & 7 & 0.00047 & 0.054 \\
response to oxidative stress & 11 & 0.00049 & 0.05 \\
response to oxygen levels & 9 & 0.0018 & 0.11 \\
response to hypoxia & 8 & 0.0043 & 0.17 \\
response to decreased oxygen levels & 8 & 0.0051 & 0.19 \\
regulation of oxidative stress-induced cell death & 4 & 0.0063 & 0.21
\end{tabular}

Data shows highly significant association of miR-210 with selected GO classifications related to hypoxia and oxidative stress, mediated through various target genes of miR-210. Benjamini value represents corrected $\mathrm{P}$-value for multiple hypothesis testing using the Benjamini-Hochberg method to minimise false discovery rate. 

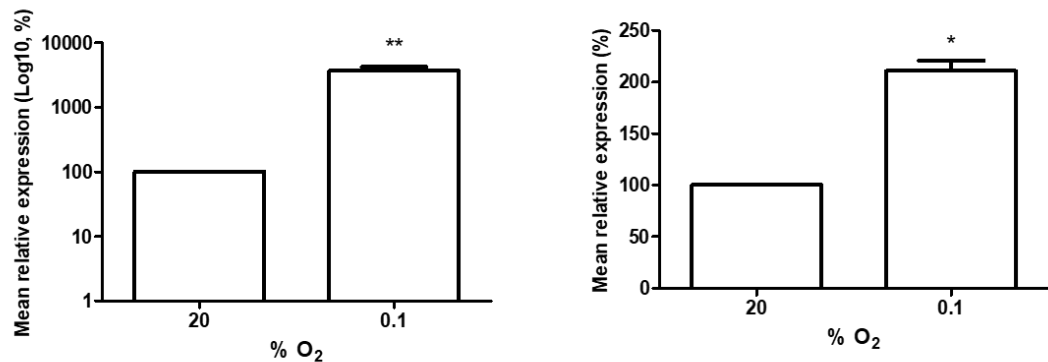

RWPE1, after $48 \mathrm{~h}$

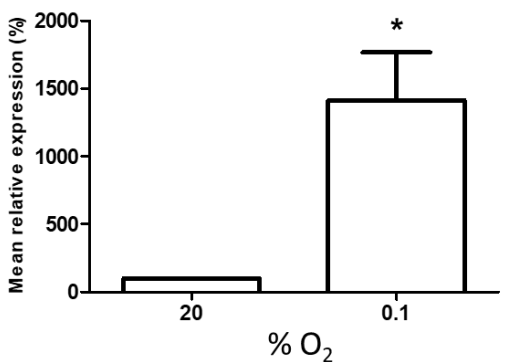

Supplementary Figure 1. miR-210 expression in hypoxic 22Rv1, PC3 and RWPE1 cells.

qRT-PCR analysis showed increased expression of miR-210 in 22Rv1, PC3 and RWPE1 cells exposed to hypoxia. PCR data was normalised to either HPRT and data shown is mean \pm SE of triplicate experiments. (Student t-test $p$-values: ${ }^{*} p<0.05,{ }^{* *} p<0.01,{ }^{* * *} p<0.001$ ). 
A

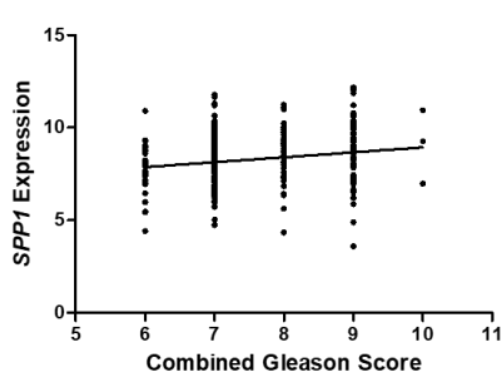

B

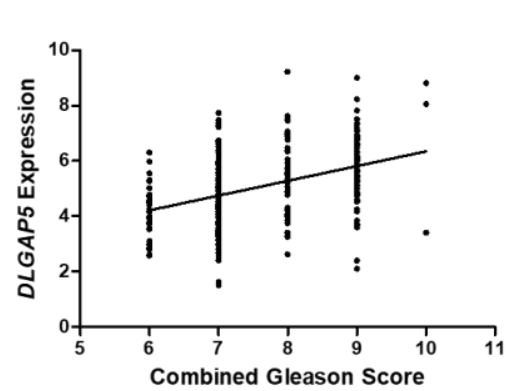

Adjusted $-\log 10(p$-value $)=3.2$ Correlation $=0.19$ $\mathrm{N}=333$

Adjusted $-\log 10$ (p-value $)=12.3$ Correlation $=0.38$ $\mathrm{N}=333$
SPP1 correlation with clinic-pathological features

\begin{tabular}{lcccc}
\hline & \multicolumn{5}{c}{$\begin{array}{c}\text { Spearman's } \\
\text { Clinical Feature }\end{array}$} & Samples $\boldsymbol{N}$ & $\begin{array}{c}\text { Correlation } \\
\text { p-value }\end{array}$ & Q-value \\
\hline $\begin{array}{l}\text { Gleason score } \\
\text { Number of lymph }\end{array}$ & 497 & 0.2134 & $1.59 \mathrm{E}-06$ & $1.44 \mathrm{E}-05$ \\
nodes with & & & & \\
metastasis & 406 & 0.1627 & 0.001 & 0.0115 \\
Pathology T stage & 490 & 0.1454 & 0.001246 & 0.0075 \\
PSA value & 440 & 0.1726 & 0.000274 & 0.00679
\end{tabular}

DLGAP5 correlation with clinic-pathological features

\begin{tabular}{|c|c|c|c|c|}
\hline Clinical Feature & Samples $N$ & $\begin{array}{l}\text { Spearman's } \\
\text { Correlation }\end{array}$ & p-value & Q-value \\
\hline $\begin{array}{l}\text { Gleason score } \\
\text { Number of lymph } \\
\text { nodes with }\end{array}$ & 496 & 0.4251 & $3.51 \mathrm{E}-23$ & $1.65 \mathrm{E}-20$ \\
\hline metastasis & 405 & 0.211 & $1.86 \mathrm{E}-05$ & 0.000859 \\
\hline Pathology T stage & 489 & 0.3312 & $5.59 \mathrm{E}-14$ & 2.69E-11 \\
\hline PSA value & 439 & 0.1538 & 0.001231 & 0.0155 \\
\hline
\end{tabular}

\section{Supplementary Figure 2. Expression of SPP1 and DLGAP5 in clinical prostate biopsies.}

(A) Regulome analysis of one TCGA dataset $(n=330)$ shows SPP1 expression is significantly correlated with Gleason score. Firebrowse analysis of TCGA prostate biopsy samples showing positive correlation of SPP1 with clinic-pathological parameters of disease progression. (B) Regulome analysis of one TCGA dataset $(n=330)$ shows DLGAP5 expression is significantly correlated with Gleason score. Firebrowse analysis of TCGA prostate biopsy samples showing positive correlation of DLGAP5 with clinic-pathological parameters of disease progression. TCGA analyses utilised Spearman's rank correlation, with $p$-values adjusted for multiple hypothesis testing. A $p$-value of $<0.05$ and $Q$-value $<$ 0.3 was deemed significant. 


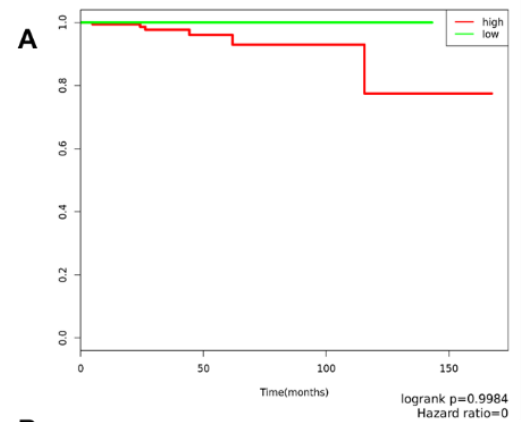

C

B
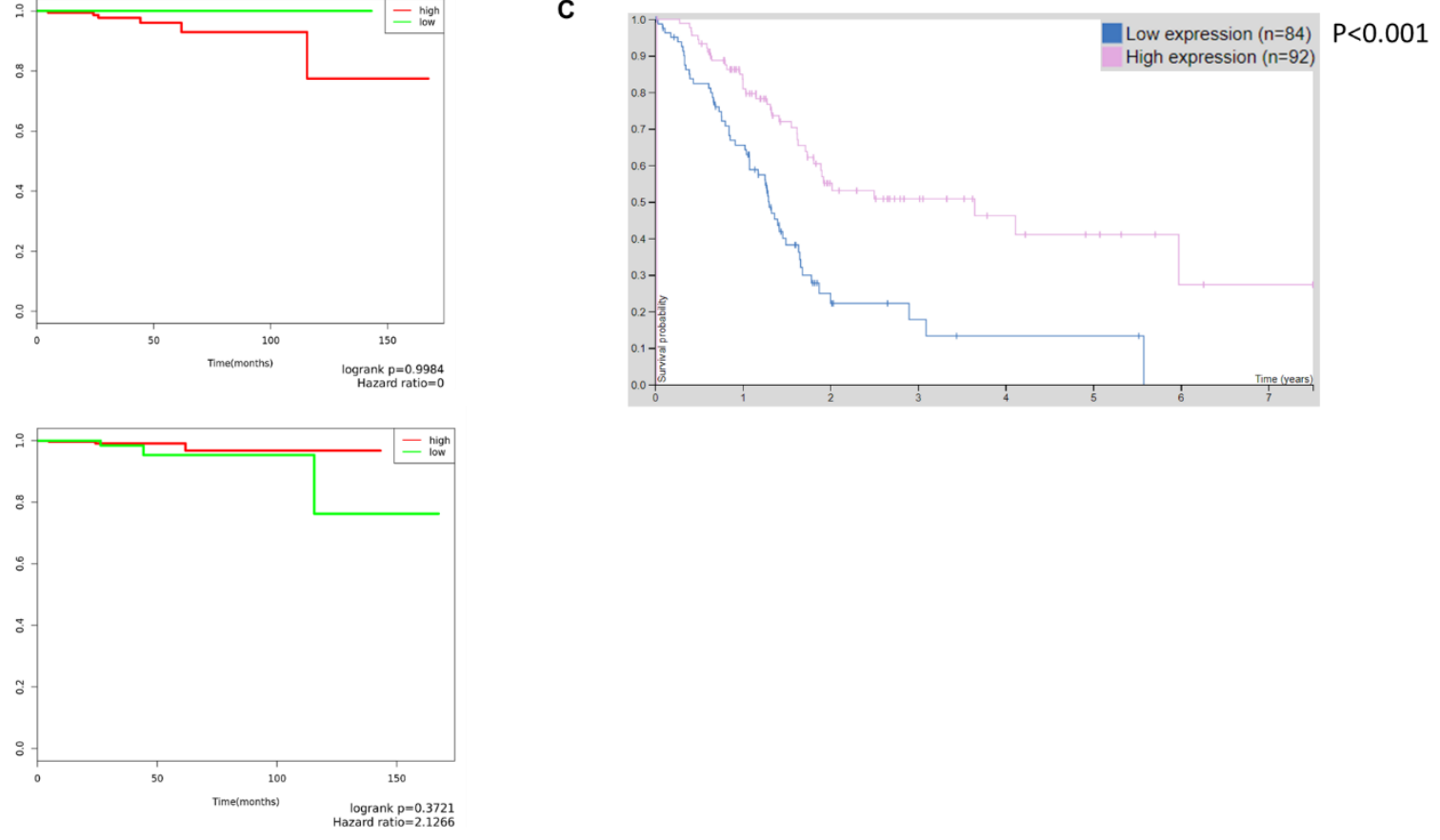

Supplementary Figure 3. Survival analysis

(A) KM Express survival analysis of NCAM1 expression shows it is not significantly associated with lower 5-year survival in prostate cancer. (B) However, Human Protein Atlas survival analysis shows lower NCAM1 expression is associated with significantly shorter 5-year survival probability in pancreatic cancer. (image credit: Human Protein Atlas. image/gene/data available from v18.1. www.proteinatlas.org . Data available at https://www.proteinatlas.org/ENSG00000149294NCAM1/pathology/tissue/pancreatic+cancer [Accessed 08-02-2019]) 


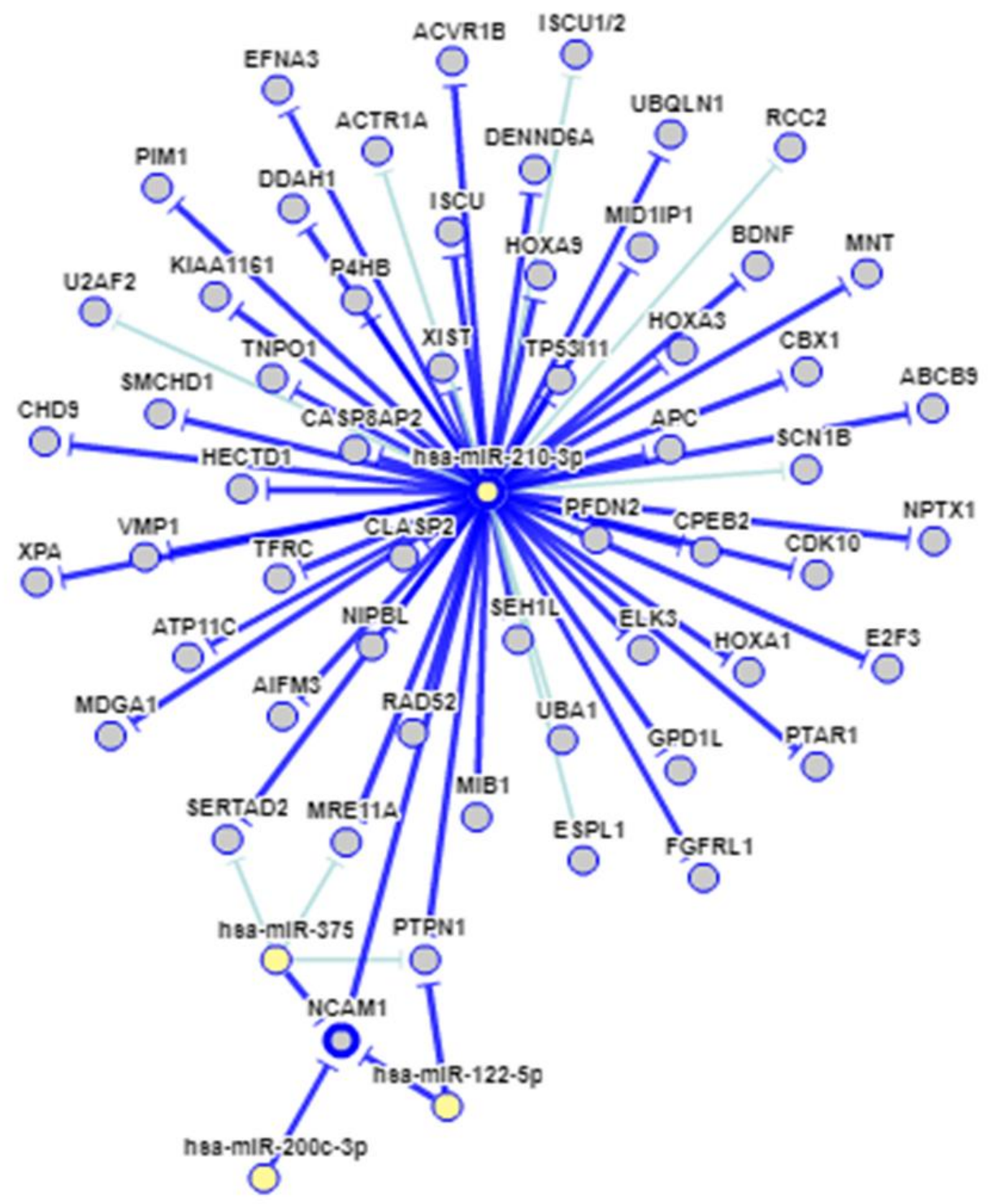

\section{Supplementary Figure 4. miR-210 Regulatory network}

Network analysis using the miRTarbase website (http://mirtarbase.mbc.nctu.edu.tw/index.php) reveals that miR-210 impacts upon several other targets. Likewise, NCAM1 is regulated by other miRNAs. Accession ID: MIRT003159 [miRNA, hsa-miR-210-3p :: NCAM1, target gene] 

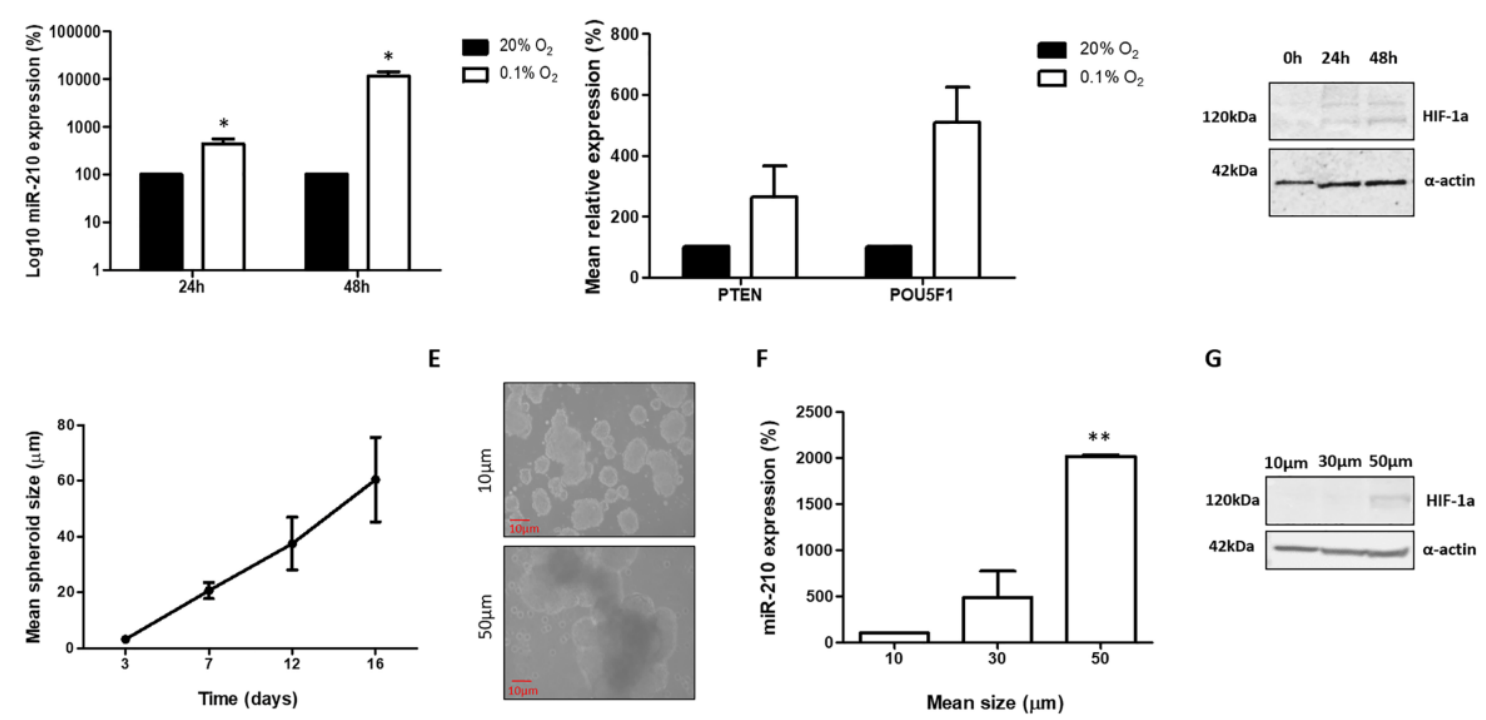
A

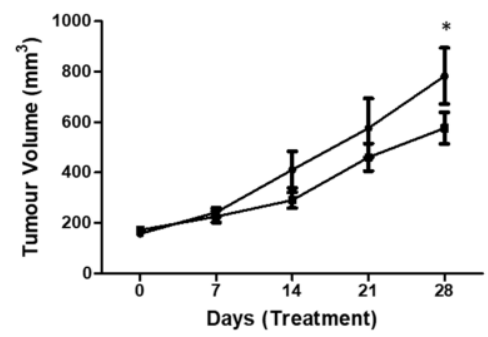

C

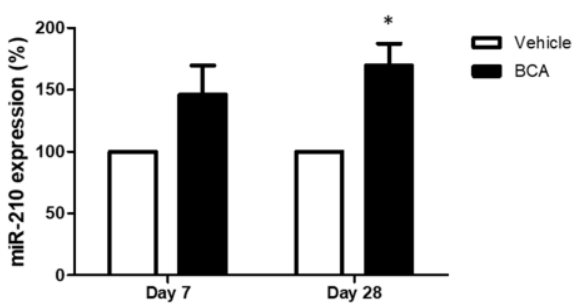

B

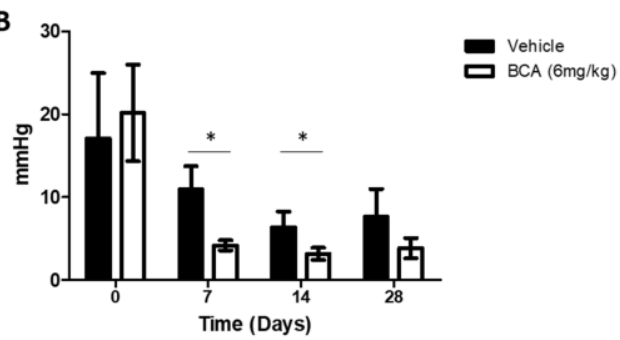

E

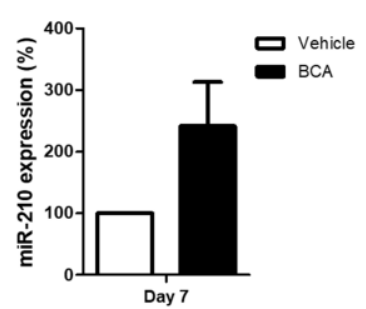

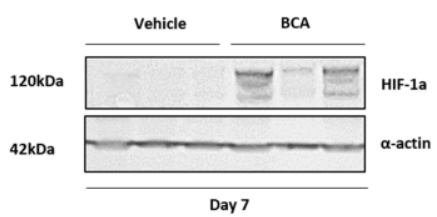


A

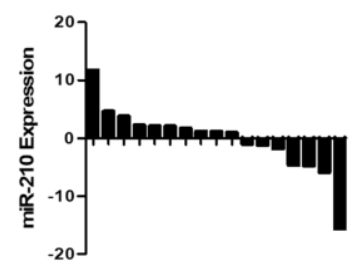

D

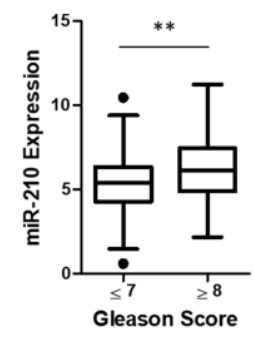

B

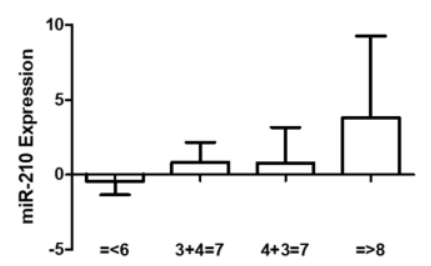

E

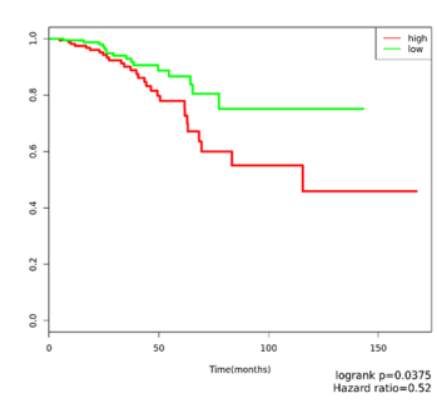

C
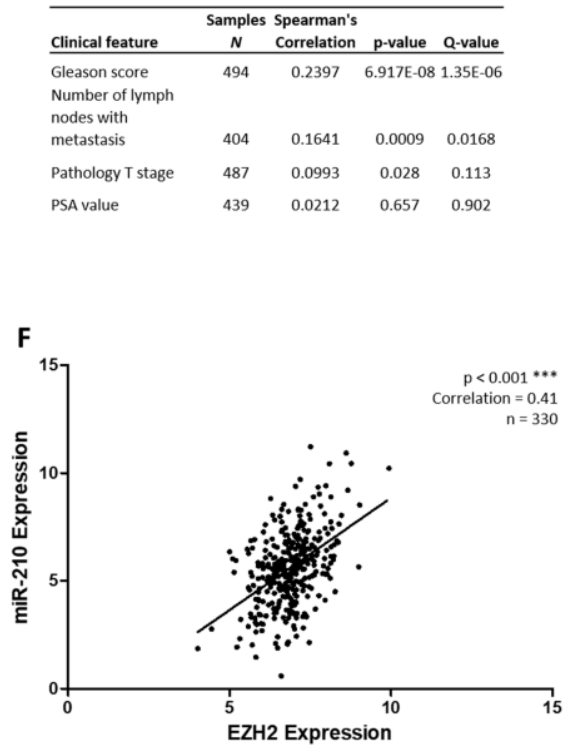
A

miR-2103' agucggcgacaGUGUGCGUGUC NCAM15' agacacacacaCGCACGCACAC

miR-2103' agUC-GGCGAC--AGUGUGCGUGUc NCAM15' acAGATCTCTGGTTCAAATGCACAg

miR-2103' agucggcgacaGUGUGCGUGUC

NCAM15' acacacaaacaCACATGCACACAC

D

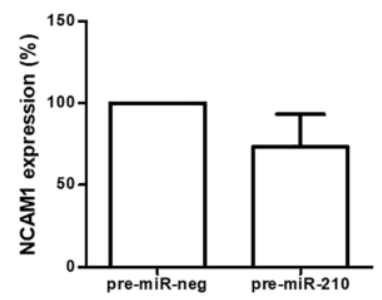

B

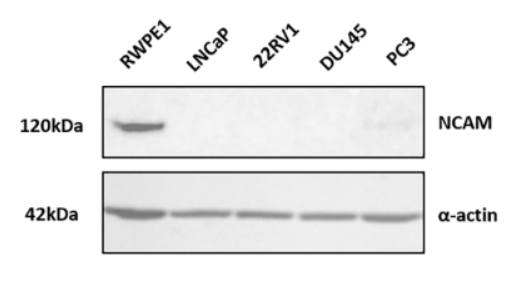

E

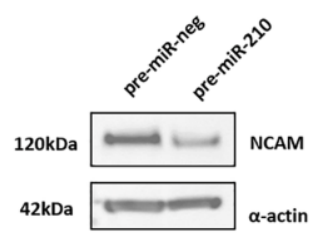

C

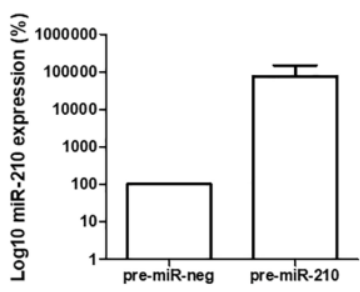

$\mathbf{F}$

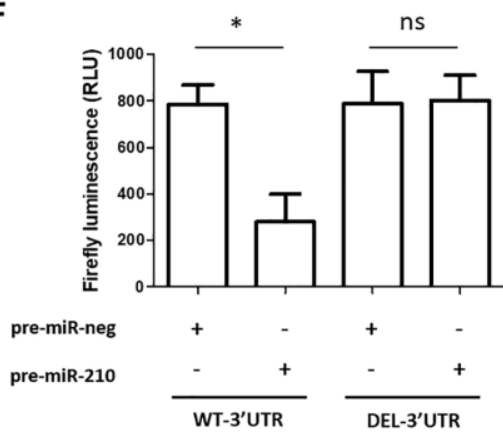


A

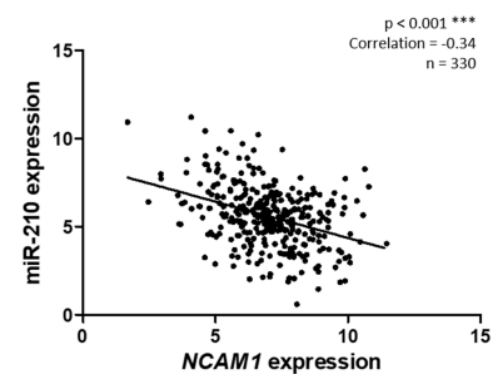

B

\begin{tabular}{lcccc}
\hline & \multicolumn{5}{c}{$\begin{array}{c}\text { Spearman's } \\
\text { Clinical Feature }\end{array}$} & Samples $\boldsymbol{N}$ & Correlation & p-value & Q-value \\
\hline Gleason score & 497 & -0.1282 & 0.004215 & 0.0128 \\
$\begin{array}{l}\text { Number of lymph } \\
\text { nodes with }\end{array}$ & & & & \\
metastasis & 406 & -0.1282 & 0.009714 & 0.0531 \\
Pathology T stage & 490 & -0.0414 & 0.3601 & 0.522 \\
PSA value & 440 & -0.0211 & 0.6593 & 0.809
\end{tabular}

C

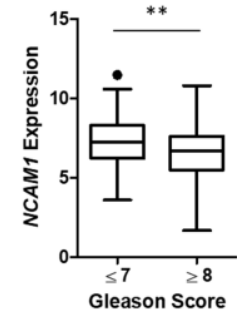

